\title{
Light trapping by arbitrarily thin cavities
}

\author{
Tilek Zhumabek (1) and Constantinos Valagiannopoulos (ํ) \\ Department of Physics, School of Sciences and Humanities, Nazarbayev University, 53 Qabanbay Batyr Ave, \\ Nur-Sultan KZ-010000, Kazakhstan
}

(Received 19 June 2020; revised 21 October 2020; accepted 16 November 2020; published 9 December 2020)

\begin{abstract}
Simple plasmonic multilayers are found to perfectly trap electromagnetic waves around specific incidence directions, regardless of the sample thickness. Such a counterintuitive feature is acquired via increasing unboundedly the effective path length of the ray into the film with proper tilt of the optical axis. The obtained optimal setups support wide-band operation while being substantially robust with respect to fabrication defects. Accordingly, the reported designs may exceptionally serve a range of objectives, from photovoltaic conversion and sensing to light modulation and detection, as parts of photonic devices requiring high efficiency combined with compact packaging.
\end{abstract}

DOI: 10.1103/PhysRevResearch.2.043349

\section{INTRODUCTION}

Light trapping, namely, capturing the incoming photons and confining them into a volume, is a fundamental operation behind numerous photonic applications from sensing, and detection to optical tagging and photoelectrochemistry [1]. A major mechanism to collect the incident illumination is via electromagnetic absorption whose operational principles and rules of design have been formulated [2]. In particular, nanometer-thin optical coatings making resonant cavities are shown to completely absorb the propagating rays [3] while metamaterial unit cells are proposed for coupling separately to electric and magnetic fields so as to trap all incident radiation within a single layer [4]. Following an alternative design route, active media making conjugately matched layers are introduced to soak up all the available power via both propagating and evanescent fields acting as ideal sinks for electromagnetic energy [5]. The metasurface paradigm is adopted in the fabrication of golden nanodisks constituting an infrared perfect absorber for refractive index sensing [6] and in the isolation of graphene nanoribbons able to trap photons for dynamically tunable light modulation [7]. Blackbody, a theoretical material that absorbs all incoming light, has been also modeled with media of hyperbolic dispersion [8] or radially inhomogeneous texture [9] and has been fabricated by vertically aligned single-walled carbon nanotubes [10].

By far the most popular application for these ultraefficient absorbing and trapping effects is the design of solar cells and

\footnotetext{
${ }^{*}$ Corresponding author: konstantinos.valagiannopoulos @ nu.edu.kz

Published by the American Physical Society under the terms of the Creative Commons Attribution 4.0 International license. Further distribution of this work must maintain attribution to the author(s) and the published article's title, journal citation, and DOI.
}

panels for photovoltaic use. More specifically, silicon wire arrays [11] or clusters of silver "islands" [12] offer opportunities to manipulate the ratio of illumination area over solar absorption volume and reduce the materials' consumption while a simple and scalable method to fabricate them has been also implemented [13]. Alternatively, ultrathin films supporting admittance matching may lead to optimal layered absorbers [14], and two-dimensional monolayers are found to hold untapped potential for thermal conversion at the nanoscale [15]. In a similar way, textured transparent conductive oxides are shown to realize highly functioning light-trapping schemes. Importantly, conventional limits [16] for the performance of photovoltaic cells are reported to be surpassed when optical modes exhibit deep-subwavelength-scale field confinement [17] or by designing an elevated local density of optical states for the absorber [18].

This vast applicability potential of light-trapping and absorption phenomena has inevitably ignited (and fed by) significant funding initiatives. In particular, National Science Foundation (NSF) has recently supported programs on experimentation to enhance power conversion efficiency, and exploration of scale-up processes for low-cost perovskite solar cells [19] as well as research projects on the development of next-generation panels for clean energy operation [20]. In addition, NSF has approved proposals for the transformational impact on perfect light absorption in the visible spectrum via machine-learning-guided density functional theory [21] while the US Office of Naval Research (ONR) is sponsoring research efforts that combine cutting-edge approaches in synthesis of state-of-the-art materials capable of generating electricity from sunlight [22]. Most interestingly, several Multi-University Research Initiatives (MURIs), by US Department of Defense (DoD), are currently running on near-field radiative energy conversion in nanogaps of metastructured media [23] and on enhancing thermal transport at material interfaces [24].

In this work, we demonstrate almost perfect absorption of incoming light, by films comprising free-standing tilted 


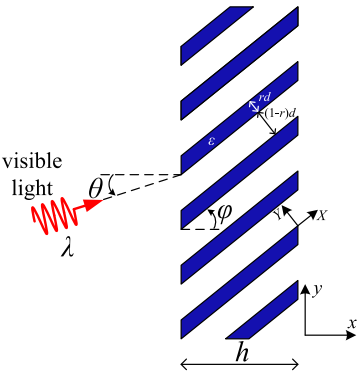

(a)

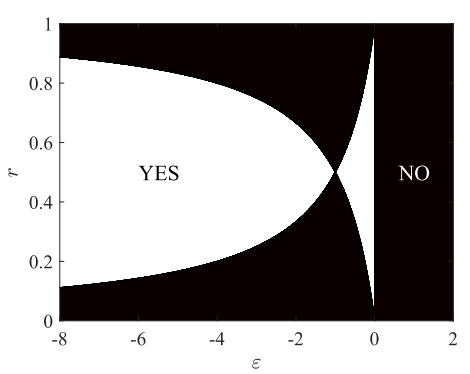

(b)
FIG. 1. (a) Schematic of the proposed configuration: an obliquely incident plane wave oscillating with wavelength $\lambda$ meets under angle $\theta$ a uniaxial cavity comprised of free-standing multilayers of duty cycle $r$ and tilted optical axis by angle $\varphi$, filled by a medium of relative complex permittivity $\varepsilon=\varepsilon(\lambda)$. The primary $(x, y, z)$ and the auxiliary $(X, Y, z)$ coordinate systems are also defined. (b) Existence map of the resonance leading to perfect absorption regime, according to Eqs. (5) and (7), for a spectrum of permittivity $\varepsilon$ and material volume fraction $r$.

multilayers. Our intention is to achieve such a flawless result regardless of the cavity thickness; thus, we select the parameters of the film so that the effective path length into the slab increases unboundedly. Analytical conditions for that regime concerning the tilt of the optical axis and the incident angle are derived; they demand materials with plasmonic behavior [25,26] able to shrink the effective wavelengths. Based on these constraints, ordinary metals with realistic losses are employed and the absorbance is maximized. The reported optimal designs support wide-band operation while their light-trapping performance exhibits substantial robustness with respect to engineering defects and is practically independent from the size of the cavity. Our approach differs from other studies of similar purpose utilizing the band structure of cubic photonic crystal [27], Floquet-Bloch waves into graphene nanoflakes [28], or waveguiding within short parallel plates [29]. When it comes to the fabrication of the cavities, there are several methods for stacking multiple layers at the nanoscale, both chemical [30] and physical [31] or even self-assembling techniques [32], accompanied by lithographic liftoff [33] for properly cutting the samples. To sum up, the proposed designs possess a series of advantages since they are simultaneously (i) thickness indifferent, even ultrathin, (ii) highly absorbing, (iii) feasible to fabricate, and (iv) robust to engineering imperfections. Therefore, they can constitute indispensable pieces of integrated systems related to light trapping like solar panels, optical sensors, and photonic modulators [34].

\section{MATHEMATICAL FORMULATION}

\section{A. Boundary value problem}

We consider the photonic setup depicted in Fig. 1(a), where the used coordinate system $(x, y, z)$ is also defined. A plane wave of visible light with wavelength $\lambda$ meets obliquely under angle $\theta$ a slab of thickness $h$. The planar film is comprised of multilayers with period $d \ll \lambda$; each layer has size $r d<d$ and is filled by a homogeneous material of relative permittivity $\varepsilon$.
The multilayers are free standing with no loss of generality while being cut with their local $X$ axis forming an angle $\varphi$ with $x$ direction. The effective permittivities along their major axes $(X, Y)$ are given by $\varepsilon_{X}=(1-r)+\varepsilon r$ and $\varepsilon_{Y}=\frac{\varepsilon}{(1-r) \varepsilon+r}$, based on well-known homogenization formulas [35]; thus, the permittivity tensor $[\varepsilon]$ in the global Cartesian coordinate system $(x, y, z)$ takes the form [36]

$$
\begin{aligned}
{[\varepsilon] } & =\left[\begin{array}{ccc}
\varepsilon_{x x} & \varepsilon_{x y} & 0 \\
\varepsilon_{x y} & \varepsilon_{y y} & 0 \\
0 & 0 & \varepsilon_{X}
\end{array}\right] \\
& =\left[\begin{array}{ccc}
\varepsilon_{X} \cos ^{2} \varphi+\varepsilon_{Y} \sin ^{2} \varphi & \left(\varepsilon_{X}-\varepsilon_{Y}\right) \cos \varphi \sin \varphi & 0 \\
\left(\varepsilon_{X}-\varepsilon_{Y}\right) \cos \varphi \sin \varphi & \varepsilon_{Y} \cos ^{2} \varphi+\varepsilon_{X} \sin ^{2} \varphi & 0 \\
0 & 0 & \varepsilon_{X}
\end{array}\right] .
\end{aligned}
$$

We assume that the incident electric field lies on the xy plane so that it "feels" a nonisotropic structure and, given the two-dimensional nature of the setup, the magnetic field is parallel to the $z$ axis everywhere. If the incident magnetic field is of unitary magnitude and written as $\mathbf{H}_{\text {inc }}=\hat{\mathbf{z}} e^{-i k_{0}(x \cos \theta+y \sin \theta)}$, the reflective and transmissive ones take the forms $\mathbf{H}_{\mathrm{ref}}=\hat{\mathbf{z}} R \boldsymbol{e}^{-i k_{0}(-x \cos \theta+y \sin \theta)}$ and $\mathbf{H}_{\mathrm{tran}}=$ $\hat{\mathbf{z}} T e^{-i k_{0}(x \cos \theta+y \sin \theta)}$, respectively, all into free space. The symbol $k_{0}=2 \pi / \lambda$ is used for the wave number into vacuum and the suppressed time dependence is of the form $\exp \left(+i k_{0} c t\right)$, where $c$ is the speed of light into free space. The reflection and transmission coefficients $\{R, T\}$ are determined as follows, by imposing the necessary boundary conditions at the interfaces of the film:

$$
\begin{aligned}
& R=\frac{2 i \sin (k h)\left(u^{2}-k^{2} \varepsilon_{x x}^{2}\right)}{e^{+i k h}\left(u+k \varepsilon_{x x}\right)^{2}-e^{-i k h}\left(u-k \varepsilon_{x x}\right)^{2}}, \\
& T=\frac{4 e^{i k_{0} h\left(\cos \theta+\frac{\varepsilon_{x y}}{\varepsilon_{x x}} \sin \theta\right)} u k \varepsilon_{x x}}{e^{+i k h}\left(u+k \varepsilon_{x x}\right)^{2}-e^{-i k h}\left(u-k \varepsilon_{x x}\right)^{2}},
\end{aligned}
$$

where $u=k_{0}\left(\varepsilon_{x x} \varepsilon_{y y}-\varepsilon_{x y}^{2}\right) \cos \theta$ and $k$ is given by

$$
k=k_{0} \sqrt{\left(\varepsilon_{x x}-\sin ^{2} \theta\right)\left(\frac{\varepsilon_{y y}}{\varepsilon_{x x}}-\frac{\varepsilon_{x y}^{2}}{\varepsilon_{x x}^{2}}\right)} .
$$

\section{B. Perfect trapping conditions}

In order for the device depicted in Fig. 1(a) to absorb $100 \%$ of the incoming illumination of specific angle $\theta$ and wavelength $\lambda$, its front interface should be matched with free space, namely, inflict zero reflections. By inspection of (2), it is clear that such a demand $(R=0)$ is feasible by nullifying one of the two factors in the numerator of reflection coefficient $R$. However, since we aim at reporting a thickness-independent effect, we avoid perfect matching with Fabry-Perot [37] interference (first factor) and, thus, we set equal to zero the second factor $\left(u^{2}-k^{2} \varepsilon_{x x}^{2}\right)$, namely,

$$
\begin{aligned}
\cos ^{2} \theta & =\frac{-1+\varepsilon_{Y}+\left(\varepsilon_{X}-\varepsilon_{Y}\right) \cos ^{2} \varphi}{-1+\varepsilon_{X} \varepsilon_{Y}} \\
\Rightarrow \cos ^{2} \theta & =\frac{1-(1-\varepsilon)(1-r) \cos ^{2} \varphi}{1+\varepsilon} .
\end{aligned}
$$


Similar constraints are obtained for metasurfaces of cylindrical rods with use of equivalent moments [38]. Given the fact that some losses $\operatorname{Im}[\varepsilon]<0$ are necessary for absorption, (5) cannot lead to real angles $\theta \in \mathbb{R}$. Nonetheless, the concept described in this study works also for very small losses $\operatorname{Im}[\varepsilon]$ and we can assume that the material in this analytical approach is almost lossless. To put it alternatively, the derived formulas correspond to perfect trapping of the incident light and, in the presence of some losses, to significant absorption of it. In the latter case, $|\operatorname{Im}[\varepsilon]|$ should not be zero giving absorption $A=0$ nor of significant magnitude that will make a complex $\theta$ according to (5); that is why one may say that an ideal absorbing scenario would occur for a medium with $0 \neq \operatorname{Im}[\varepsilon] \rightarrow 0^{-}$.

But, how may it be possible for an almost lossless medium to absorb $100 \%$ of the incident light and, most importantly, within a very small volume of $k_{0} h$ tending to zero? The answer lies in increasing the length of the path that the wave is following into the cavity. In particular, the magnetic field into the film is written in the form [39] $\mathbf{H}_{\text {film }}=$ $\hat{\mathbf{z}} e^{-i k_{0} y \sin \theta}\left[U e^{-i \kappa_{+} x}+V e^{-i \kappa_{-} x}\right]$, where

$$
\kappa_{ \pm}=-\frac{\varepsilon_{x y}}{\varepsilon_{x x}} k_{0} \sin \theta \pm k,
$$

$\{U, V\}$ are determinable complex constants, and $k$ is defined by (4). If one manages to increase unboundedly one (or both) of the wave numbers $\kappa_{ \pm}$into the slab, the length of the corresponding path will also blow up. Both the quantities $\kappa_{ \pm}$ have the same denominator which vanishes for a tilt angle $\varphi$ satisfying

$$
\cos ^{2} \varphi=\frac{-\varepsilon}{(1-\varepsilon)^{2}(1-r) r},
$$

valid again for $\operatorname{Im}[\varepsilon] \rightarrow 0^{-}$. The condition (7) makes the element $\varepsilon_{x x}$ from (1) equal to zero; it also leads to a vanishing transmission from (3) which, combined with zero reflection (2) due to (5), gives $100 \%$ absorption. To put it more strictly, even when $\operatorname{Im}[\varepsilon]=0$ one can achieve a simultaneous nullification of $R$ and $T$ by managing to create waves that travel "forever" into the slab (trapping regime), namely, to support infinite wave numbers $\kappa_{ \pm}$. It should be noted that, since $0<r<1$, (7) is only possible for plasmonic media with $\varepsilon<0$.

By combining the two conditions (5) and (7), we obtain an explicit expression for the incoming direction as $\cos ^{2} \theta=$ $\frac{\varepsilon+(1-\varepsilon) r}{\left(1-\varepsilon^{2}\right) r}$. Most importantly, the wave numbers into the film take the following form:

$$
\frac{k_{0}}{\kappa_{ \pm}} \sim \cos \varphi\left[\cos \varphi \pm \frac{1}{1-\varepsilon} \sqrt{\frac{-\varepsilon}{(1-r) r}}\right],
$$

where the behaviors of the two quantities $\left\{\kappa_{+}, \kappa_{-}\right\}$from (6) are interchangeable in proportion to the sign of the parameters $\{\theta,(\varepsilon+1)\}$. It should be stressed that if (7) applies, one of the two wave numbers in Eq. (8) tends to infinity while the other remains finite. Such a result verifies the condition (7) and will lead to perfect trapping within an arbitrarily thin film. Indeed, there will be no reflections due to (5) and the wave entering the cavity will cover a huge, due to (7) and (8), path during which will convert all its power in thermal form, even in the presence of slight nonzero $\operatorname{Im}[\varepsilon]$. It must be remarked that the exact

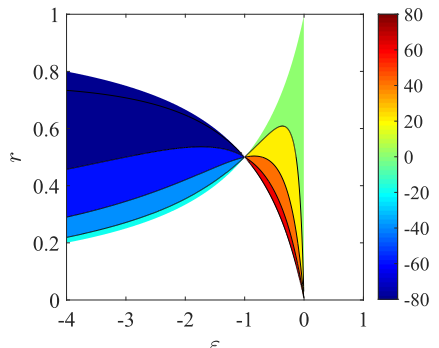

(a)

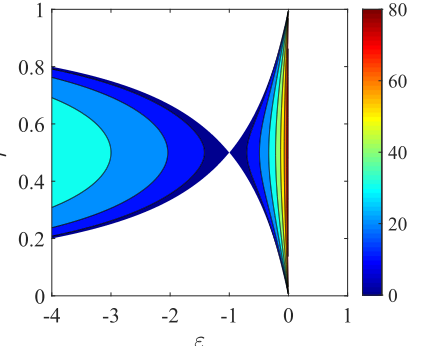

(b)
FIG. 2. Variation of the optimal angles leading to thicknessindependent perfect absorption with respect to (real part of) relative permittivity $\varepsilon$ and duty cycle $r$. (a) The incidence angle $\theta$ (in degrees) from (5). (b) The tilt angle $\varphi$ (in degrees) from (7). Results only for the feasible combinations according to Fig. 1(b) are depicted.

mapping of the modes $\kappa_{ \pm}$to the limiting forms of (8) is not of prime importance since both modes are activated into the finite-thickness slab. We do not care which of the two waves goes infinitely fast; either way (towards $+x$ or towards $-x$ ), the incoming ray will be forced to travel through an extremely long path into the film.

In Fig. 1(b), we show which combinations of permittivities $\varepsilon$ and material volume factors $r$ can satisfy simultaneously (5) and (7) with real angles $(\theta, \varphi)$; the acceptable parametric pairs are presented with white color (region labeled as "YES") and the infeasible ones with black color (region labeled as "NO"). One notices that two allowable areas are formulated touching each other at a single point $(\varepsilon, r)=\left(-1, \frac{1}{2}\right)$; the first one concerns dielectric constants with $-1<\varepsilon<0$ and the more one approaches epsilon-near-zero (ENZ) regime, much more extensive $r$ ranges are available. The second area corresponds to deeply negative permittivities $\varepsilon<-1$ and once they become lower, the permissible duty cycle $r$ interval gets mildly increased. As indicated by (7), the infinite wave-number regime, which guarantees the infinitely long path for the wave into the film, is only possible for plasmonic media $(\varepsilon<0)$; such a feature is also shown in the map of Fig. 1(b).

Every single couple $(\varepsilon, r)$ able to host the perfect trapping effect decides a pair of angles $(\theta, \varphi)$ containing an incoming direction $-90^{\circ}<\theta<90^{\circ}$ and an optical axis tilt $0^{\circ}<\varphi<$ $90^{\circ}$. In Fig. 2(a), we show the variation of angle $\theta$ on the $(\varepsilon, r)$ map wherever the conditions (5) and (7) are applicable, namely, across the white region of Fig. 1(b). It is remarkable that angles referring to $\varepsilon<-1$ are always negative contrary to those assuming $-1<\varepsilon<0$ for which $\theta>0$; in this way, two distinct resonance regimes are identified. In the former domain, the incoming wave should advance more obliquely as $r$ increases at a fixed $\varepsilon<-1$. As far as the latter domain is concerned, the trend is opposite: a closer to normal ray is required to get trapped. In Fig. 2(b), we represent the change of the inclination $\varphi$ across $(\varepsilon, r)$ plane and realize that it is an even function with respect to $r=\frac{1}{2}$ line. Importantly, more tilted axes are required for $-1<\varepsilon<0$ as one moves towards ENZ materials and for $\varepsilon<-1$ as the medium gets deeper and deeper plasmonic. Finally, as the difference of $r$ from $\frac{1}{2}$ increases, the required tilt of the major axes into the multilayered structure becomes lower. 


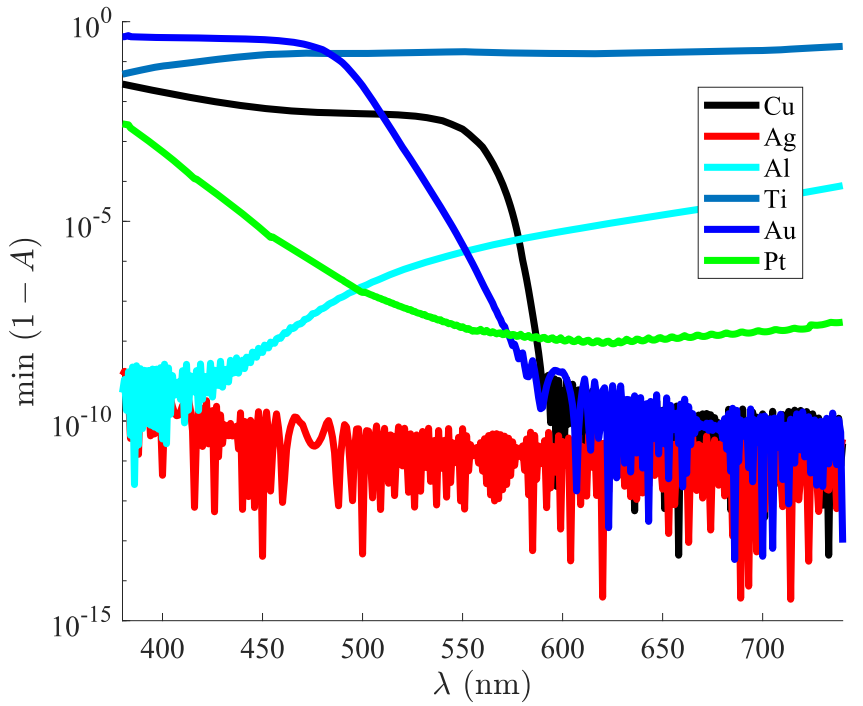

FIG. 3. The minimum nonabsorbed power $(1-A)$ with respect to duty cycle $r$, according to our approximate analytical optimization, as a function of operational wavelength $\lambda$ for various metals with dispersive permittivity $\varepsilon=\varepsilon(\lambda)$. In all cases, it happens to be $\operatorname{Re}[\varepsilon(\lambda)]<-1$. The selection of angles is made by satisfying the conditions (5) and (7) for each specific $\lambda$ and assuming that $\operatorname{Im}[\varepsilon]=0$. Thickness: $h=100 \mathrm{~nm}$.

\section{NUMERICAL RESULTS}

\section{A. Optimized absorbing designs}

Once the permittivity $\varepsilon$ is well known, namely, if one fixes the operational wavelength $\lambda$ and the employed material with its dispersive formula $\varepsilon=\varepsilon(\lambda)$, a permissible interval for duty cycle $r$ is defined according to Figs. 1(b) and 2. Across this $r$ range, there are suitable real $(\theta, \varphi)$ from (5) and (7) leading to total trapping of light. However, the absorbance $A$ is not always unitary, as the model predicts, since the losses $\operatorname{Im}[\varepsilon]<0$ are non-negligible. For this reason, optimization with respect to $r$ can be performed in order to decide what are the best incidence angles $-90^{\circ}<\theta<90^{\circ}$ and multilayer tilts $0^{\circ}<\varphi<90^{\circ}$ giving the highest absorption $A$. In Fig. 3, we show the variation of the maximal performance of the device, in terms of the nonabsorbed power $(1-A)$, as function of visible-light wavelength $\lambda$, where the materials of permittivity $\varepsilon$ are some of the most ordinary metals. We are using metals and not dielectrics or semiconductors with $\operatorname{Re}[\varepsilon]>0$, since a plasmonic behavior is required to give a real angle $\varphi \in \mathbb{R}$ from (7); the evaluation of the angles is made according to (5) and (7), by ignoring the imaginary parts of $\varepsilon$. At each wavelength $\lambda$, we pick the design $\{r, \theta, \varphi\}$ that exhibits the highest absorption $A$ for a specific thickness $h=100 \mathrm{~nm}$, whose value is not playing a crucial role; in this sense, Fig. 3 comprises curves of optimal points referring to different configurations as $\lambda$ changing.

It is noteworthy that silver, the less lossy metal, shows extreme absorbance for all the wavelengths of visible spectrum; in the antipode, titanium, with its huge $|\operatorname{Im}[\varepsilon]|$, does not absorb significantly at any point of the considered frequency band. It is an anticipated outcome since the analytical model, summarized by the constraints (5) and (7), works perfectly for vanishing imaginary part of $\varepsilon$; the more the losses $|\operatorname{Im}[\varepsilon(\lambda)]|$, the less successful is the selection of the angles $(\theta, \varphi)$ in terms of achieving unitary absorbance $A$. In addition, aluminum prefers violet light to absorb contrary to designs made of gold and copper that trap the red light, as dictated by the dispersion of their $\operatorname{Im}[\varepsilon(\lambda)]$; the permittivity data are taken from reliable sources $[40,41]$ containing experimental measurements and not by employing the semianalytical Drude model. It is noted that the different trend of the curve referring to aluminum-based setups, compared to all the others, may be attributed to its deeply plasmonic behavior at short visible-light wavelengths. As far as platinum-air multilayers are concerned, they absorb more effectively the orange incoming light, even though their $A$ is kept at a remarkably low level across almost the entire regarded spectrum. If the metallic strips were not free standing but hosted in a dielectric slab of the same dimension (thickness $h$ ), the maximal absorbing performance achieved is not expected to be substantially lower than this indicated by Fig. 3. Indeed, the scattering field from the multilayers should be modified in order to neutralize the new background field (incident wave on a homogeneous dielectric slab) so that there is no aggregate reflection and, simultaneously, make a null overall transmission [42].

It should be stressed that the incidence angle, which is not shown in the graph, takes a specific value $\theta \cong-45^{\circ}$ at the optimal regime (when $A \rightarrow 1$ ), while the tilt direction of the optical axis into the cavity is almost normal to the incoming ray: $\varphi \cong 45^{\circ}$; such a feature has been also met in similar structures serving alternative objectives like polarization engineering [43]. In Fig. 4, we try to interpret the negative value of $\theta$ leading to high absorbance $A$. When the two angles defining incidence $\theta$ and tilt $\varphi$ are of the same sign and especially if $\theta=\varphi$ as in Fig. 4(a), the incident ray does not interact much with the lossy plasmonic layers; thus, the thermal losses are low and the transmission large. On the contrary, if $\theta=\varphi-90^{\circ}<0$, the incoming light meets normally the metallic flakes and is forced to follow a huge path into the film due to the successive reflections being, theoretically, infinite in number; therefore, the transmissivity almost vanishes. We must point out that the aforementioned condition, properly combined with (5) and (7), yields to

$$
\theta=\varphi-90^{\circ} \Rightarrow \cos ^{2} \theta=\sin ^{2} \varphi \underset{(5)}{\stackrel{(7)}{\Rightarrow}} \varepsilon=1-\frac{2}{r},
$$

requiring $\varepsilon<-1$ since $0<r<1$. In other words, it refers to the area of deeply plasmonic permittivities of Fig. 1(b) and thus the reflections in Fig. 4(b) are almost perfect; indeed, they occur between media with substantial textural contrast.

When it comes to the optimal value of ratio $r$, it is low and decreases with $\lambda$, regardless of the material and its losses' dependence on frequency. By inspection of Fig. 3, we can understand that there are multiple realistic designs reaching unitary performance $A$ across most wavelengths of the visible, and clearly the proposed concept following the analytical approach of Sec. II B is validated. Note, finally, that all the considered metals are deeply plasmonic $\operatorname{Im}[\varepsilon(\lambda)]<-1$ across the investigated band; therefore, all the shown optimal cases concern the first type of resonances of Figs. 1(b) and 2 which exist for $\theta<0$ and are interpreted by the ray tracing schema of Fig. 4. 


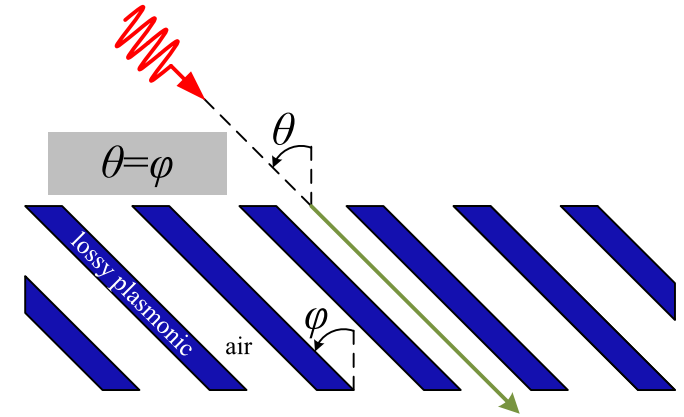

(a)

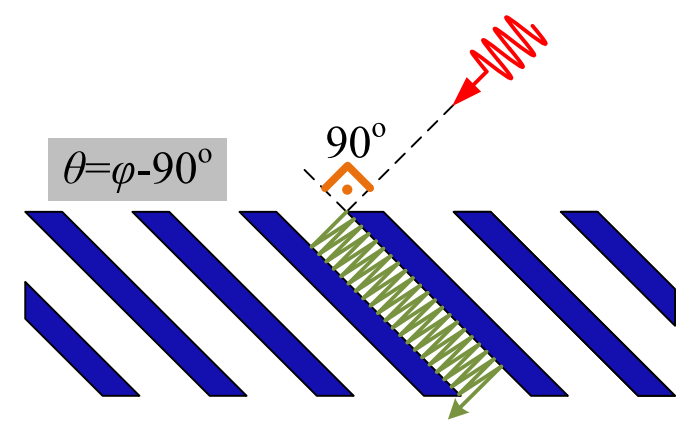

(b)

FIG. 4. Heuristic interpretation of the perfect light-trapping regime. Approximate route of a ray of light for (a) $\theta=\varphi$ (minimal trapping), (b) $\theta=\varphi-90^{\circ}$ (maximal trapping). The condition between angles of incidence and tilt $\theta=\varphi-90^{\circ}$ validates the constraints (5) and (7) as long as $\operatorname{Re}[\varepsilon]<-1$. The successive reflections in Fig. 4(b) are considered as perfect due to the large textural mismatch between air and the deeply plasmonic lossy material.

To understand better the signal distribution across the considered setup, in Fig. 5 we represent the logarithm of the magnetic field magnitude $\log \left|H_{z}\right|$ as function of the horizontal distance $x$ normalized by the operational wavelength $\lambda$ for the two different types of materials $(\operatorname{Re}[\varepsilon]<-1$ and $-1<\operatorname{Re}[\varepsilon]<0$ ) indicated by Figs. 1(b) and 2. In Fig. 5(a), we consider an incidence angle $\theta$ opposite to that securing maximal trapping [we call this regime as "minimal trapping" and is $\theta=45^{\circ}$ when $\operatorname{Re}[\varepsilon]<-1$, as indicated in Fig. 4(a)]. In the deeply plasmonic scenario, we observed an unperturbed variation into the homogenized slab leading to a full transmission of the signal, as predicted by Fig. 4(a). On the contrary, when $-1<\operatorname{Re}[\varepsilon]<0$ there are some moderate losses across the slab while the level of reflection is kept extremely low. In Fig. 5(b), we consider a beam that meets normally $\left(\theta=0^{\circ}\right)$ our structure; one directly notices that there is a substantial impedance mismatch at the front boundary $x=0$ creating a standing wave pattern for $x<0$. As far as the behavior within the slab is concerned, a rapid fall is noticed allowing for a very tiny transmission at $x>h$, especially if $\operatorname{Re}[\varepsilon]<-1$.

Finally, in Fig. 5(c), the maximal trapping effect is demonstrated showing that almost zero reflection and transmission is achieved, regardless of the type of material $\varepsilon$. It is noted that, due to the extremely small values of the nonabsorbed power $(1-A)$, we used software of increased accuracy to evaluate the respective formulas. In addition, the exponential decrease

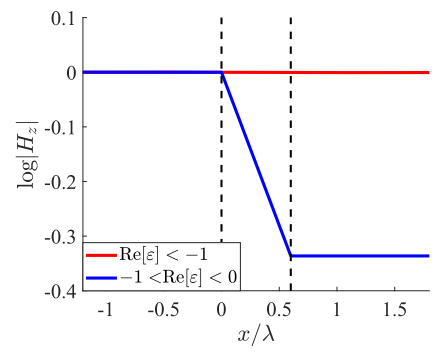

(a)

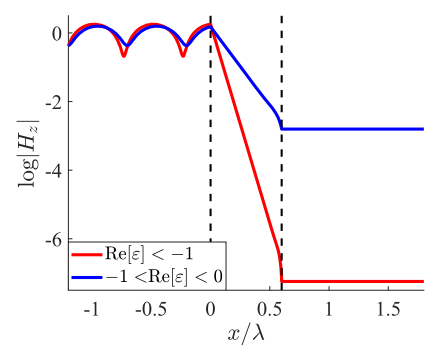

(b)

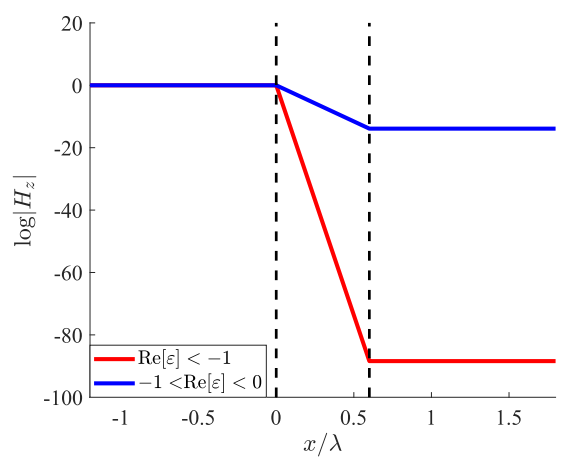

(c)

FIG. 5. The logarithm of sole magnetic field magnitude $\log \left|H_{z}\right|$ as a function of the horizontal (normal to the boundaries) coordinate $x$ for (a) $\theta$ giving minimal trapping, (b) $\theta=0^{\circ}$, (c) $\theta$ giving maximal trapping for each type of material $\varepsilon=-10-0.25 i$ (red) and $\varepsilon=-0.6-0.1 i$ (blue). Plot parameters: $h=300 \mathrm{~nm}$. The black dashed lines denote the boundaries of the slab, while the rest of the parameters $(r, \varphi)$ have been optimized to maximize overall absorption in Fig. 5(c).

into the cavity means that only one of the two modes possesses nonzero amplitude while perfect matching occurs at $x=0$. Figure 5(c) also shows that the trapping regime is practically independent from the thickness $h$ since the decline of the field $\left|H_{z}\right|$ is so abrupt that two to three orders of magnitude signal reduction happens within the first nanometers.

In Fig. 6, we pick four designs using different metals and working optimally at different wavelengths: aluminum based at $\lambda=400 \mathrm{~nm}$, silver based at $\lambda=500 \mathrm{~nm}$, platinum based at $\lambda=600 \mathrm{~nm}$, and gold based at $\lambda=700 \mathrm{~nm}$ and we examine their absorption when the characteristics of the excitation (wavelength $\lambda$, angle $\theta$ ) are changing. Note that all the points in the contours of Fig. 6 refer to the same configuration, namely, the same multilayers $(r, \varphi)$ optimized at the point indicated by "o"; the only changing part is the incoming light. One clearly observes that the light-trapping effect is very resilient with respect to $\lambda$ in all cases and especially when aluminum [Fig. 6(a)] or platinum [Fig. 6(c)] is incorporated in the design. Note that the less lossy silver exhibits the least wide-band absorption; that is because the optimal features of its setup (especially $r$ and $\varphi$ ) and its complex permittivity $\varepsilon$ is changing significantly with $\lambda$. As far as the angle $\theta$ is concerned, the trapping hits the peak at $\theta \cong-45^{\circ}$ and retains the large value $A \cong 1$ across a considerable angular extent for $\theta<0$ which becomes larger for increasing $\lambda$; on the contrary, the absorption $A$ drops dramatically for $\theta>0$ and almost gets 


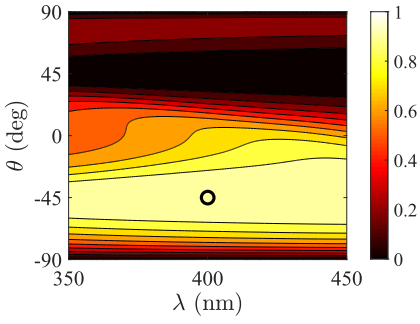

(a)

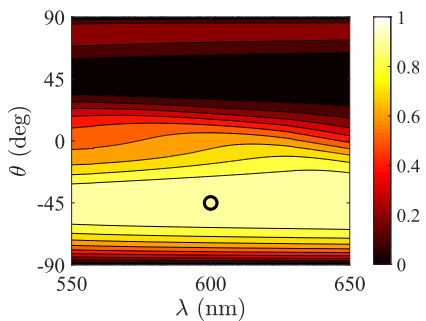

(c)

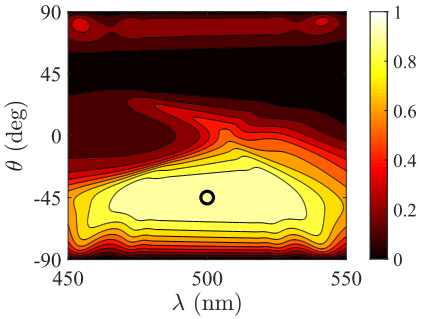

(b)

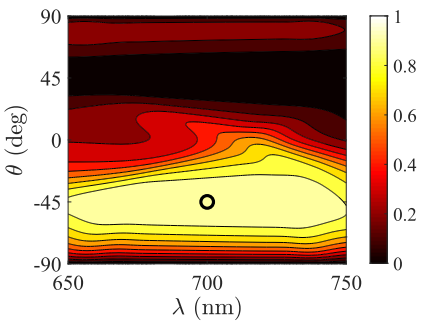

(d)
FIG. 6. Behavior of optimal design at the "source spectra plane." The absorption power $A$ is represented as a function of the wavelength $\lambda$ (color) and the angle $\theta$ (direction) of the incoming ray; the rest of parameters $(r, \varphi)$ are kept equal to their ideal values. Different materials constituting the multilayers are employed: (a) aluminum $(\lambda=400 \mathrm{~nm})$, (b) silver $(\lambda=500 \mathrm{~nm})$, (c) platinum $(\lambda=600 \mathrm{~nm})$, (d) gold $(\lambda=700 \mathrm{~nm})$ at wavelength ranges that each of which delivers best. The optimal regimes are denoted by "०." Again, the selection of angles $(\theta, \varphi)$ is made by satisfying the conditions (5) and (7) and assuming that $\operatorname{Im}[\varepsilon]=0$. Thickness: $h=100 \mathrm{~nm}$.

nullified for $\theta \cong 45^{\circ}$. Such observations are perfectly compatible with the sketches of Fig. 4; indeed, for $\theta=\varphi \cong 45^{\circ}$ [Fig. 4(a)], the ray "sees" am almost transparent structure, avoids to interact with the lossy layers, and is not absorbed by the film. Oppositely, when $\theta=-\varphi \cong 45^{\circ}$ [Fig. 4(b)], the incoming wave meets almost normally the plasmonic layers and gets trapped between a pair of them via reflecting a huge number of times; as a result, it exhausts its power and gives $A \rightarrow 1$ (perfect light trapping).

\section{B. Robustness to fabrication defects}

The multilayered structure of Fig. 1(a) can be fabricated by adopting both chemical and physical approaches. Chemical vapor deposition (CVD) exploits chemical reactions to build successive layers [30]; a subset of this group of methods is atomic layer deposition (ALD), a sequential technique where reactive media are sprayed by other substances (precursors) to generate stacked coatings $[44,45]$. Another fabrication category of planar multilayers consists of the physical depositions and especially molecular beam epitaxy (MBE), where a heated substrate gets shot molecule-by-molecule with effusion cells containing the required media in gas form $[31,46]$. Even self-organized routes are available for building multilamellar layouts of plasmonic eutectics, in combination with suitable micropulling [32]. When it comes to the cutting of the samples, various lithographic processes are available [33,47] where the liftoff can be performed with high accuracy. In most of the aforementioned methodologies, the deposition of layers with thickness much smaller than the oscillation wavelength $\lambda$ is possible; thus, the homogenization assumption for $d \ll \lambda$ [with reference to Fig. 1(a)], yielding the approximate formulas for $\varepsilon_{X}, \varepsilon_{Y}$, is justified. One may point out that the fabrication of such a layered setup is challenging when working at the visible frequencies where all the metals exhibit plasmonic behavior. However, the constraint for $\operatorname{Re}[\varepsilon]<0$ can be easily fulfilled at much longer wavelengths by ordinary dielectrics [48].

Alternatively, the required anisotropy expressed through the permittivity tensor $[\varepsilon]$ from (1) can be directly achieved by employing nanoparticles with similar effective properties. Such a synthesis [49] constitutes a seed-mediated growth procedure, in which metal salts are reduced initially with a strong reducing agent, in water, to produce the desired seed particles. In addition, anisotropic wetting processes [50] can be implemented to give directional properties to thin slabs, extensively employed in engineered uniaxial surfaces and associated applications. Furthermore, compression molding to create a thickness contrast pattern in a thin resist film carried on a substrate, followed by anisotropic etching to transfer the pattern through the entire resist thickness has been utilized, allowing fabrication of sub-10-nm structures [51]. Finally, the proposed configuration of Fig. 1(a) can be obtained with use of two-dimensional materials with built-in anisotropy, like phosphorene [52] with artificial plasmonic properties or graphene [53], fabricable via a variety of methods from mechanical exfoliation and liquid-phase preparation (top down) to wafer-scale synthesis and epitaxial growth (bottom up).

However, an extremely high resolution should not be taken for granted and, thus, it is important to see how optimized designs (like the four ones of Fig. 6 in Sec. III A) deliver if the multilayers are imperfectly grown (wrong $r$ ) or mistakenly cut (wrong $\varphi$ ). In Fig. 7, we witness the relatively high robustness of the proposed designs. In particular, all the cavities absorb almost $100 \%$ of the incoming photonic rays even though the tilt of the strips deviates more than $10^{\circ}$ from the optimal; furthermore, they are more sensitive to changes of $r$ towards smaller values than to bigger ones. The most robust setup is the Al-based one, which is also the least eager to change with respect to $(\lambda, \theta)$ according to Fig. 6(a); on the contrary, the other absorber that supports wide band $A$ in Fig. 6(c) (Pt-based) does a worse job in the presence of fabrication defects. It should be finally noticed that the cavities employing the most commonly used metals [Ag in Fig. 7(b) and Au in Fig. 7(d)] see their performance $A$ dropped dramatically when the angle $\varphi$ is chosen very different from the operation point.

A basic advantage of the proposed setups is that the trapping of light is practically independent of the thickness $h$ of the slab. Indeed, since (one of) the wave numbers $\kappa_{ \pm}$increases infinitely according to (7) and (8), all the absorption can happen even at very small $h$. To demonstrate such a feature, and also to test the robustness of the structures in Fig. 8, we represent $A$ as a function of $h$ for the designs of Figs. 6 and 7 , optimized for $h \cong 100 \mathrm{~nm}$. A large range of thicknesses from $1 \AA$ to $1 \mu \mathrm{m}$ has been considered and we realize that most of the curves surpass the threshold of $90 \%$ if the size is as tiny as a few nanometers; this finding justifies our claim that the indicated light trapping may occur at arbitrarily thin 


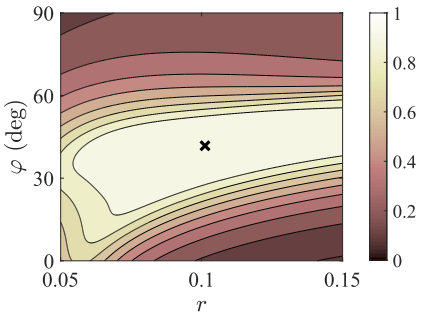

(a)

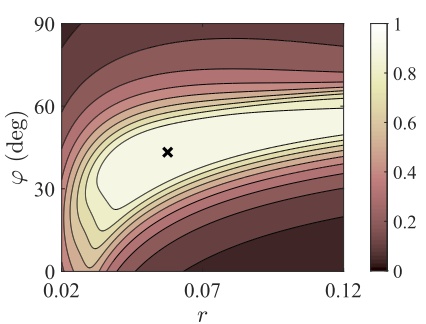

(c)

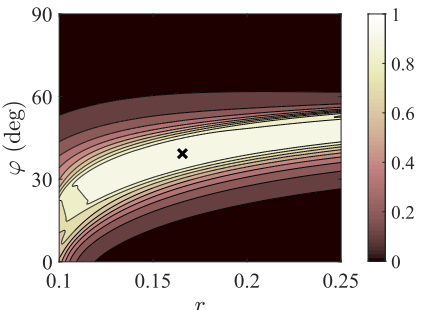

(b)

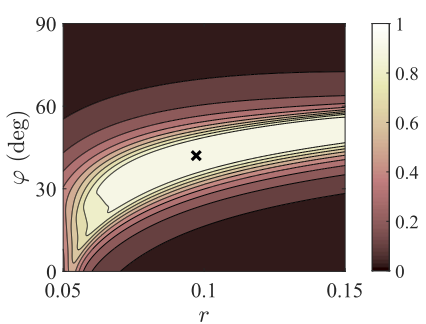

(d)
FIG. 7. Behavior of optimal designs of Fig. 6 at the "fabrication defects plane." The absorption power $A$ is represented as a function of the duty cycle $r$ and tilt of the optical axis $\varphi$; the rest of parameters $(\lambda, \theta)$ are kept equal to their ideal values. Different materials constituting the multilayers are employed: (a) aluminum $(\lambda=400$ $\mathrm{nm})$, (b) silver $(\lambda=500 \mathrm{~nm})$, (c) platinum $(\lambda=600 \mathrm{~nm})$, (d) gold $(\lambda=700 \mathrm{~nm})$. The optimal regimes are denoted by " $\times$." Thickness: $h=100 \mathrm{~nm}$.

cavities. It should be stressed that the reported scores $A$ for $h<100 \mathrm{~nm}$ can be further improved if the design $\{\lambda, \theta, r, \varphi\}$ is optimized for that smaller thickness of the film. The most remarkable performance is recorded for Ag-air multilayers where the design works flawlessly for $h>2 \mathrm{~nm}$ but, given the fact that a minimum realistic thickness is around $10 \mathrm{~nm}$,

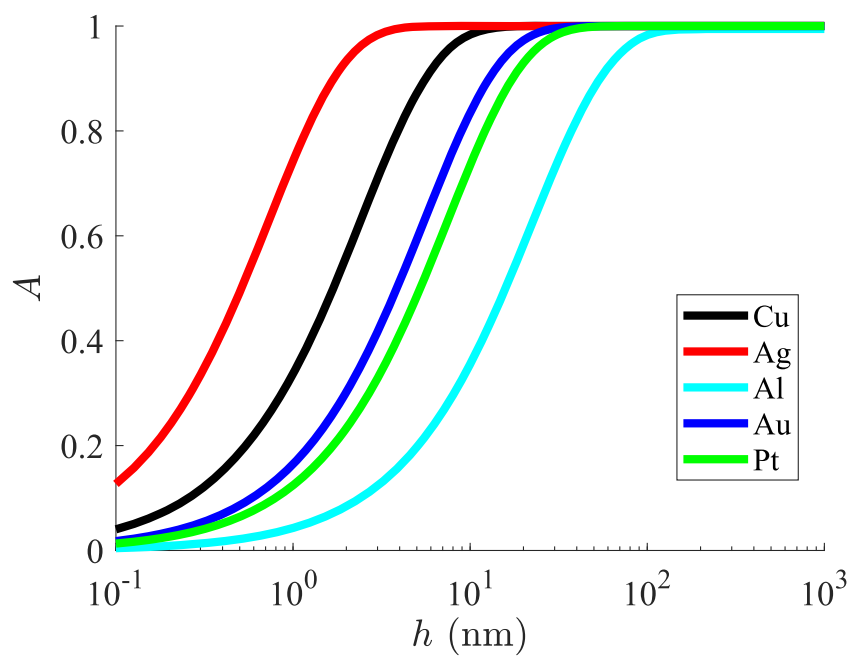

FIG. 8. The absorbance $A$ for the designs of Figs. 6 and 7 plus the Cu-based one optimized at $\lambda=650 \mathrm{~nm}$ and when $h=100 \mathrm{~nm}$ from Fig. 3 as function of the sample thickness $h$. Thickness-independent trapping is demonstrated since $A$ takes almost unitary values for cavities thicker than a few nanometers.

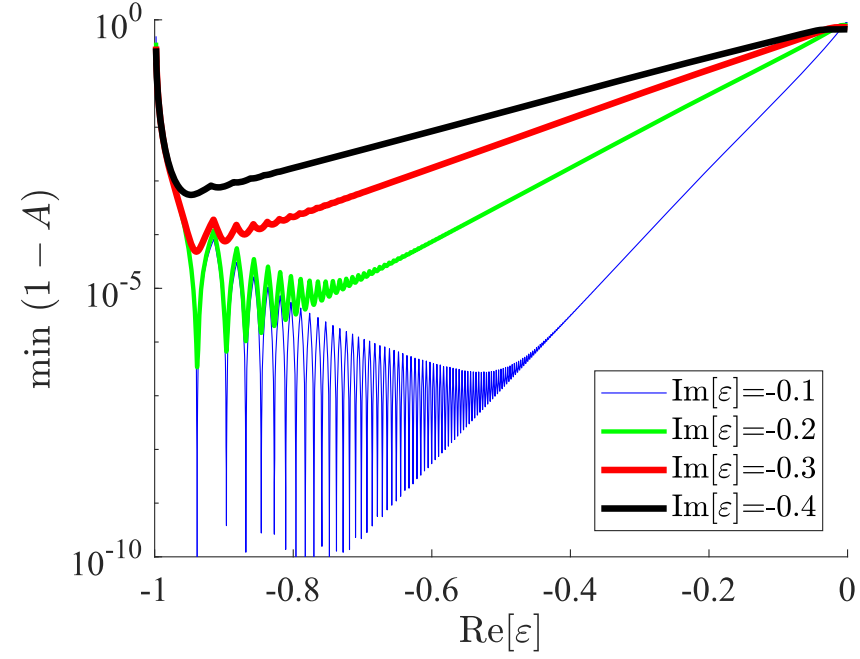

FIG. 9. The minimum nonabsorbed power $(1-A)$ with respect to duty cycle $r$, according to our approximate analytical optimization (5) and (7) and by assuming $\operatorname{Im}[\varepsilon]=0$, as a function of the real part of relative permittivity $-1<\operatorname{Re}[\varepsilon]<0$ for several imaginary parts $\operatorname{Im}[\varepsilon]$. The permittivity $\varepsilon=\operatorname{Re}[\varepsilon]+i \operatorname{Im}[\varepsilon]$ is considered dispersionless across the visible part of the wavelength spectrum. Plot parameters: $h=100 \mathrm{~nm}, \lambda=560 \mathrm{~nm}$.

all the cavities absorb extremely high portions of the incident field practically regardless of $h$.

\section{Case of $-1<\operatorname{Re}[\varepsilon]<0$}

All the previous examples elaborated in Secs. III A and III B concern metals that are plasmonic in the visible range; however, it happens, across the entire visible range $380 \mathrm{~nm}<$ $\lambda<740 \mathrm{~nm}$, that their permittivities have real parts with $\operatorname{Re}[\varepsilon(\lambda)]<-1$. In other words, all the cases that have been investigated in the presented results concern only one of the two regions of Fig. 1(b) where the perfect light trapping becomes feasible. Therefore, in this section we will consider a fictitious dispersionless medium with $-1<\operatorname{Re}[\varepsilon]<0$ and observe how their multilayers absorb the incoming illumination under the well-known conditions (5) and (7). It should be noted that plasmonic materials with low $|\operatorname{Re}[\varepsilon]<0|$ can be found at a variety of oscillating wavelengths $\lambda$. In particular, near-zero negative real permittivity has been experimentally achieved with use of refractory conductors in far-ultraviolet [54], by constructing nanodisk arrays at optical frequencies [55] or via the employment of ultranarrow waveguide channels operated with radio waves [56]. In Fig. 9, we show an analog of Fig. 3 for that case of nondispersive dielectric constant $-1<\operatorname{Re}[\varepsilon(\lambda)]<0$. In particular, we represent the nontrapped power $(1-A)$ as function of the real part $\operatorname{Re}[\varepsilon]$ of permittivity for various level of losses $\operatorname{Im}[\varepsilon]$. As in Fig. 3, we have minimized the quantity $(1-A)$ with respect to duty cycle $0<r<1$; once again, the angles $(\theta, \varphi)$ are determined via the analytical formulas (5) and (7), by assuming $\operatorname{Im}[\varepsilon]=0$. The thickness of the sample is taken equal to that of Figs. 3 and $6-8$, namely, $h=100 \mathrm{~nm}$ while the operational wavelength is kept fixed to, approximately, the mean along the visible spectrum $(\lambda=560 \mathrm{~nm})$. We define a wavelength even though the used material is nondispersive since the thickness 


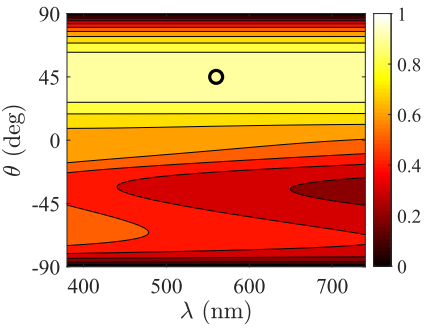

(a)

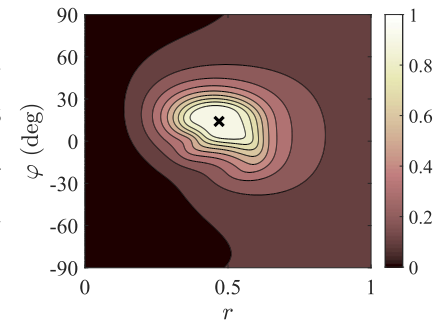

(b)

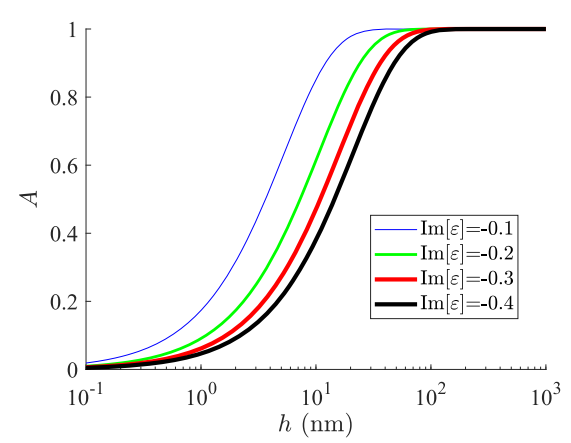

(c)

FIG. 10. (a) The absorption power $A$ as a function of the source features; the optimal regime is denoted by "०" and $\varepsilon=-0.6-0.1 i$. (b) The absorption power $A$ as a function of the fabrication defects; the optimal regime is denoted by " $x$ " and $\varepsilon=-0.6-0.1 i$. (c) The absorbance $A$ for the designs of Fig. 9 when $h=100 \mathrm{~nm}$ as function of the sample thickness $h$; again thickness-independent trapping is indicated, like in Fig. 8. Plot parameters: $h=100 \mathrm{~nm}, \lambda=560 \mathrm{~nm}$.

$h$ is expressed in terms of $\lambda$; however, the results would be identical for any setup with the same $h / \lambda \cong 0.18$, regardless of the oscillation frequency.

By inspection of Fig. 9, it is clear that the trapping effect is very weak when $\operatorname{Re}[\varepsilon] \rightarrow 0$ since plasmonic character is mandatory for $\varphi \in \mathbb{R}$ defined by (7), and when $\operatorname{Re}[\varepsilon] \rightarrow$ -1 because there is virtually no $r$ fitting the approximate analytical model, according to Figs. 1(b) and 2. Nonetheless, in-between these two limiting values $-1<\operatorname{Re}[\varepsilon]<$ 0 , the minimum $(1-A)$ obtains very low values, despite some negligible oscillations reminding us that every single point of the graphs in Fig. 9 is the result of an optimization. Finally, as also understood by Fig. 3, the low level of losses gives a more efficient absorption since the employed analytical model (requiring $\operatorname{Im}[\varepsilon] \rightarrow 0^{-}$) becomes more realistic.

In Fig. 10(a), we pick one of the designs investigated in Fig. $9(\varepsilon=-0.6-0.1 i)$ optimized for thickness $h=100 \mathrm{~nm}$ at wavelength $\lambda=560 \mathrm{~nm}$, and represent the absorption $A$ in contour plot on the $(\lambda, \theta)$ map. Importantly, the optimal regime occurs at a positive angle $\theta \cong 45^{\circ}$ and the operation is quite wide angle (reminding other setups [57]) since it retains the very high levels of light trapping for a range even larger than the realistic layouts in Fig. 6. Note that the heuristic schema of Fig. 4(b) does not apply in this case since the condition (9) is not respected; indeed, $\theta>0$ since $-1<$ $\operatorname{Re}[\varepsilon]<0$. The most striking feature of Fig. 10 (a) is the hugely wide-band behavior of the absorption $A$ across the entire the considered frequency interval; indeed, $h / \lambda$ plays no role because $\operatorname{Re}[\varepsilon]<0$ is kept fixed as well as the losses which are also small $(\operatorname{Im}[\varepsilon]=-0.1)$, namely, the analytical model is applied almost perfectly. In Fig. 10(b), we examine the harmful influence of the fabrication defects on the same design, something analogous to Fig. 7. This time, the results are similar to those for the metal-based structures since the robustness of the setup is high with respect to both $\varphi$ and $r$. Note that, for the considered case, the optimal $\varphi$ is smaller than $45^{\circ}$ while high trapping scores are observed even for negative tilts of the optical axis $(\varphi<0)$.

In Fig. 10(c), we regard four of the designs of Fig. 9 optimized for thickness $h=100 \mathrm{~nm}$ and produce the analogous graph of Fig. 8, namely, we show the variation of absorption $A$ as function of the sample thickness $h$. Once again, we notice the sigmoid shape of curves, like in Fig. 8, and it is demonstrated that the described light-trapping effect is practically thickness-independent since $A$ gets rapidly increased after some nanometers. However, one can point out that the setups with actual metallic losses of Fig. 8 require even smaller sizes $h$ for accomplishing unitary absorption compared to the fictitious and dispersionless lossy media of Fig. 10(c).

\section{CONCLUSION}

Absorption of electromagnetic waves across the visible, infrared, or ultraviolet part of the frequency spectrum is the backbone effect behind several applications dealing with light. The true challenge is to make that light trapping occur within an ultrathin cavity for design, packing, and integration purposes. In this work, we demonstrate thickness-independent perfect absorption from films comprising multilayers with tilted optical axis; it becomes feasible if the effective path length into the slab becomes infinite, namely, when the local wave number increases unboundedly. Several metals with realistic losses are tried and almost unitary efficiency is achieved for all the colors of visible light; importantly, the maximally absorbing layouts are wide band and can trap incident rays traveling across an extensive cone around the optimal direction. Furthermore, the presented samples exhibit substantial robustness to engineering defects and, of course, the performance of light trapping is extremely weakly dependent on their size. The case of dispersionless and mildly plasmonic materials is examined separately; analogous conclusions are drawn with the difference that the band of operation is practically infinite.

The reported optimal setups possess a series of assets (ultrathin, wide band, robust) that render them particularly befitted as components in photonic devices requiring smart designing and packaging. Furthermore, the manufacturing of these configurations is feasible even at very high frequencies via a variety of techniques like chemical atomic layer deposition and physical molecular beam epitaxy while the cutting can be performed with lithographic exfoliation. Thus, our results may inspire experimental efforts towards the fabrication of such compact modules able to support, within narrow volumes, a broad range of functionalities from thermal conversion and optical sensing to light modulation and detection. 


\section{ACKNOWLEDGMENTS}

This work was partially supported by Nazarbayev University Faculty Development Competitive Research Grant with project entitled "Super transmitters, radiators and lenses via photonic synthetic matter" (Grant No. 090118FD5349). Funding from Ministry of Education and Science of the Republic of Kazakhstan state-targeted Program No. BR05236454 is also acknowledged.

[1] K. C. Neuman and S. M. Block, Optical trapping, Rev. Sci. Instrum. 75, 2787 (2004).

[2] Y. Ra'di, C. R. Simovski, and S. A. Tretyakov, Thin Perfect Absorbers For Electromagnetic Waves: Theory, Design, And Realizations, Phys. Rev. Appl. 3, 037001 (2015).

[3] M. A. Kats, R. Blanchard, P. Genevet, and F. Capasso, Nanometre optical coatings based on strong interference effects in highly absorbing media, Nat. Mater. 12, 20 (2013).

[4] N. I. Landy, S. Sajuyigbe, J. J. Mock, D. R. Smith, and W. J. Padilla, Perfect Metamaterial Absorber, Phys. Rev. Lett. 100, 207402 (2008).

[5] C. A. Valagiannopoulos, J. Vehmas, C. R. Simovski, S. A. Tretyakov, and S. I. Maslovski, Electromagnetic energy sink, Phys. Rev. B 92, 245402 (2015).

[6] N. Liu, M. Mesch, T. Weiss, M. Hentschel, and H. Giessen, Infrared perfect absorber and its application as plasmonic sensor, Nano Lett. 10, 2342 (2010).

[7] S. Kim, M. S. Jang, V. W. Brar, K. W. Mauser, L. Kim, and H. A. Atwater, Electronically tunable perfect absorption in graphene, Nano Lett. 18, 971 (2018).

[8] S. A. Biehs, M. Tschikin, and P. Ben-Abdallah, Hyperbolic Metamaterials as an Analog of a Blackbody in the Near Field, Phys. Rev. Lett. 109, 104301 (2012).

[9] E. E. Narimanov and A. V. Kildishev, Optical black hole: Broadband omnidirectional light absorber, Appl. Phys. Lett. 95, 041106 (2009).

[10] K. Mizuno, J. Ishii, H. Kishida, Y. Hayamizu, S. Yasuda, D. N. Futaba, M. Yumura, and K. Hata, A black body absorber from vertically aligned single-walled carbon nanotubes, Proc. Natl. Acad. Sci. USA 106, 6044 (2009).

[11] M. D. Kelzenberg, S. W. Boettcher, J. A. Petykiewicz, D. B. Turner-Evans, M. C. Putnam, E. L. Warren, J. M. Spurgeon, R. M. Briggs, N. S. Lewis, and H. A. Atwater, Enhanced absorption and carrier collection in $\mathrm{Si}$ wire arrays for photovoltaic applications, Nat. Mater. 9, 239 (2010).

[12] S. Pillai, K. R. Catchpole, T. Trupke, and M. A. Green, Surface plasmon enhanced silicon solar cells, J. Appl. Phys. 101, 093105 (2007).

[13] E. Garnett and P. Yang, Light trapping in silicon nanowire solar cells, Nano Lett. 10, 1082 (2010).

[14] X. Fang and C. Y. Zhao, Unified analyses and optimization for achieving perfect absorption of layered absorbers with ultrathin films, Int. J. Heat Mass Transfer 111, 1098 (2017).

[15] M. Bernardi, M. Palummo, and J. C. Grossman, Extraordinary sunlight absorption and one nanometer thick photovoltaics using two-dimensional monolayer materials, Nano Lett. 13, 3664 (2013).

[16] E. Yablonovitch, Inhibited Spontaneous Emission in Solid-State Physics and Electronics, Phys. Rev. Lett. 58, 2059 (1987).

[17] Z. Yu, A. Raman, and S. Fan, Fundamental limit of nanophotonic light trapping in solar cells, Proc. Natl. Acad. Sci. USA 107, 17491 (2010).
[18] D. M. Callahan, J. N. Munday, and H. A. Atwater, Solar cell light trapping beyond the ray optic limit, Nano Lett. 12, 214 (2012).

[19] Low-Cost, Efficient Next-Generation Solar Cells for the Coming Clean Energy Revolution, NSF Award No. 1538893, PI: N. Padture, PM: C. Whitley (2015).

[20] CCI Solar Fuels, NSF Award No. 1305124, PI: H. Gray, PM: K. Covert (2013).

[21] Machine learning exploration of atomic heterostructures towards perfect light absorber and giant piezoelectricity, NSF Award No. 1719875, PI: T. Low, PM: J. Schlueter (2017).

[22] Center For Self-Assembled Organic Electronics, NSF Award No. 1539916, PI: J. Redwing, PM: Z. Ying (2015).

[23] Near-Field Radiative Heat Transfer and Energy Conversion in Nanogaps of Nano- and Meta-Structured Materials, MultiUniversity Research Initiative 2019-2024, PI: S. Reddy, University of Michigan (2019).

[24] Leveraging a New Theoretical Paradigm to Enhance Interfacial Thermal Transport in Wide Band Gap Power Electronics, Multi-University Research Initiative 2018-2023, PI: S. Graham, Georgia Institute of Technology (2018).

[25] J. Schuller, E. Barnard, W. Cai, Y. C. Jun, J. S. White, and M. L. Brongersma, Plasmonics for extreme light concentration and manipulation, Nat. Mater. 9, 193 (2010).

[26] S. N. Shirodkar, M. Mattheakis, P. Cazeaux, P. Narang, M. Soljacic, and E. Kaxiras, Quantum plasmons with optical-range frequencies in doped few-layer graphene, Phys. Rev. B 97, 195435 (2018).

[27] B. J. Frey, P. Kuang, M.-L. Hsieh, J.-H. Jiang, S. John, and S.-Y. Lin, Effectively infinite optical pathlength created using a simple cubic photonic crystal for extreme light trapping, Sci. Rep. 7, 4171 (2017).

[28] I. S. Nefedov, C. A. Valagiannopoulos, and L. A. Melnikov, Perfect absorption in graphene multilayers, J. Opt. 15, 114003 (2013).

[29] C. A. Valagiannopoulos and S. A. Tretyakov, Emulating hyperbolic-media properties with conventional structures, New J. Phys. 16, 063004 (2014).

[30] K. Seshan, Handbook of Thin Film Deposition Processes and Techniques (William Andrew, Norwich, NY, 2001).

[31] L. N. Pfeiffer, K. W. West, R. L. Willett, H. Akiyama, and L. P. Rokhinson, Nanostructures in GaAs fabricated by molecular beam epitaxy, Bell Labs Tech. J. 10, 151 (2005).

[32] P. Osewski, A. Belardini, M. Centini, C. Valagiannopoulos, G. Leahu, R. Li Voti, M. Tomczyk, A. Alù, D. A. Pawlak, and C. Sibilia, New self-organization route to tunable narrowband

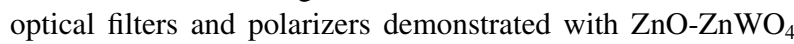
eutectic composite, Adv. Opt. Mater. 8, 1901617 (2020).

[33] K. Q. Le, Q. M. Ngo, and T. K. Nguyen, Nanostructured metalinsulator-metal metamaterials for refractive index Biosensing applications: Design, fabrication, and characterization, IEEE J. Sel. Top. Quantum Electron. 23, 388 (2017). 
[34] C. Valagiannopoulos, T. A. Tsiftsis, and V. Kovanis, Metasurface-enabled interference mitigation in visible light communication architectures, J. Opt. 21, 115702 (2019).

[35] A. Sihvola, Electromagnetic Mixing Formulas and Applications, IEEE Electromagnetic Waves Series (IEEE, Piscataway, NJ, 1999).

[36] C. A. Valagiannopoulos, Study of an electrically anisotropic cylinder excited magnetically by a straight strip line, Prog. Electromagn. Res. 73, 297 (2007).

[37] J. M. Vaughan, The Fabry-Perot Interferometer: History, Theory, Practice and Applications (Taylor \& Francis, New York, 1989).

[38] C. A. Valagiannopoulos and S. A. Tretyakov, Symmetric absorbers realized as gratings of PEC cylinders covered by ordinary dielectrics, IEEE Trans. Antennas Propag. 62, 5089 (2014).

[39] C. A. Valagiannopoulos, Controlling reflection and transmission with a single layer, IEEE Antennas Propag. Mag. 57, 318 (2015).

[40] A. D. Rakic, A. B. Djurisic, J. M. Elazar, and M. L. Majewski, Optical properties of metallic films for vertical-cavity optoelectronic devices, Appl. Opt. 37, 5271 (1998).

[41] http://refractiveindex.info/.

[42] Z. Tagay and C. Valagiannopoulos, Highly selective transmission and absorption from metasurfaces of periodically corrugated cylindrical particles, Phys. Rev. B 98, 115306 (2018).

[43] A. Sarsen and C. Valagiannopoulos, Robust polarization twist by pairs of multilayers with tilted optical axes, Phys. Rev. B 99, 115304 (2019).

[44] S. M. George, Atomic layer deposition: An overview, Chem. Rev. 110, 111 (2010).

[45] Ce-Ming Wang, De-Lin Kong, Qiang Chen, and Jian-Ming Xue, Surface engineering of synthetic nanopores by atomic layer deposition and their applications, Front. Mater. Sci. 7, 335 (2013).

[46] B. A. Joyce, Molecular beam epitaxy, Rep. Prog. Phys. 48, 1637 (1985).
[47] G. Perrakis, O. Tsilipakos, G. Kenanakis, M. Kafesaki, C. M. Soukoulis, and E. N. Economou, Perfect optical absorption with nanostructured metal films: Design and experimental demonstration, Opt. Express 27, 6842 (2019).

[48] C. A. Valagiannopoulos, N. L. Tsitsas, and A. Lakhtakia, Giant enhancement of the controllable in-plane anisotropy of biased isotropic noncentrosymmetric materials with epsilon-negative multilayers, J. Appl. Phys. 121, 063102 (2017).

[49] C. J. Murphy, T. K. Sau, A. M. Gole, C. J. Orendorff, J. Gao, L. Gou, S. E. Hunyadi, and T. Li, Anisotropic metal nanoparticles: Synthesis, assembly, and optical applications, J. Phys. Chem. B 109, 13857 (2005).

[50] D. Xia, L. M. Johnson, and G. P. López, Anisotropic wetting surfaces with one-dimesional and directional structures: Fabrication approaches, wetting properties and potential applications, Adv. Mater. 24, 1287 (2012).

[51] S. Y. Chou, P. R. Krauss, and P. J. Renstrom, Imprint lithography with 25-nanometer resolution, Science 272, 85 (1996).

[52] L. Kou, C. Chen, and S. C. Smith, Phosphorene: Fabrication, properties and applications, J. Phys. Chem. Lett. 6, 2794 (2015).

[53] L. Ju, B. Geng, J. Horng, C. Girit, M. Martin, Z. Hao, H. A. Bechtel, X. Liang, A. Zettl, Y. R. Shen, and F. Wang, Graphene plasmonics for tunable terahertz metamaterials, Nat. Nanotechnol. 6, 630 (2011).

[54] S. Kassavetis, B. D. Ozsdolay, N. Kalfagiannis, A. Habib, J.-H Tortai, S. Kerdsongpanya, R. Sundararaman, M. Stchakovsky, D. V. Bellas, D. Gall, and P. Patsalas, Near-zero negative real permittivity in far ultraviolet: Extending plasmonics and photonics with B1-MoN $x$, J. Phys. Chem. C 123, 21120 (2019).

[55] M. Habib, D. Briukhanova, N. Das, B. C. Yildiz, and H. Caglayan, Controlling the plasmon resonance via epsilon-nearzero multilayer metamaterials, Nanophotonics 9, 3637 (2020).

[56] B. Edwards, A. Alù, M. E. Young, M. Silveirinha, and N. Engheta, Experimental Verification of Epsilon-Near-Zero Metamaterial Coupling and Energy Squeezing Using a Microwave Waveguide, Phys. Rev. Lett. 100, 033903 (2008).

[57] A. N. Papadimopoulos, N. V. Kantartzis, N. L. Tsitsas, and C. A. Valagiannopoulos, Wide-angle absorption of visible light from simple bilayers, Appl. Opt. 56, 9779 (2017). 